\title{
The Study of Genetic Relationship Among Third Generation Cephalosporin-resistant Salmonella enterica Strains by ERIC-PCR
}

\author{
Reza Ranjbar $^{1}$, Ali Naghoni ${ }^{1}$, Soheila Yousefi ${ }^{2}$, Ali Ahmadi ${ }^{1, *}$, Nematollah Jonaidi ${ }^{3}$ and Yunes \\ Panahi $^{4}$ \\ ${ }^{1}$ Molecular Biology Research Center, Baqiyatallah University of Medical Sciences, Tehran, Iran \\ ${ }^{2}$ Department of Biochemistry, Payam Noor University, Tehran, Iran \\ ${ }^{3}$ Health Research Center, Baqiyatallah University of Medical Sciences, Tehran, Iran \\ ${ }^{4}$ Chemical Injuries Research Center, Baqiyatallah University of Medical Sciences, Tehran, Iran
}

\begin{abstract}
Background and Objectives: Salmonella is an important food-borne pathogen responsible for disease in humans and animals. The aim of this study was to investigate the genetic relationship among third generation cephalosporinresistant Salmonella enterica strains by Enterobacterial Repetitive Intergenic Consensus (ERIC)-PCR.

Methods: The study included all Salmonella isolates obtained from clinical cases in a pediatric hospital in Tehran, Iran during 2006 to 2009. Antimicrobial susceptibility testing was performed according to the Clinical and Laboratory Standards Institute. The genetic relationship between third generation cephalosporins-resistant Salmonella enterica strains was determined using ERIC-PCR.

Results: Of 136 Salmonella enterica isolates recovered from pediatric patients, six isolates including four Salmonella enterica serotype Infantis and two Salmonella enterica serotype Enteritidis showed an extended-spectrum cephalosporins resistant phenotype. ERIC-PCR differentiated Salmonella enterica serotypes Infantis and Enteritidis into 2 distinct clusters arbitrarily named as E1 and E2. Profile E1 was found in two Salmonella enterica serotype Enteritidis isolates, and profile E2 was found in four Salmonella enterica serotype Infantis isolates.

Conclusion: Extended-spectrum cephalosporins resistant Salmonella could be attributed to a few predominant serotypes including Enteritidis and Infantis in this study. Genetic analysis using ERIC-PCR showed that closely related clones are responsible for the occurrence of extended-spectrum cephalosporins resistant Salmonella infection in Tehran.
\end{abstract}

Keywords: Salmonella enterica, Enterobacterial Repetitive Intergenic Consensus, Antimicrobial susceptibility testing.

\section{INTRODUCTION}

Salmonella is a genus of Gram-negative rod-shaped bacteria of the family Enterobacteriaceae [1]. It has been the leading cause of many outbreaks and infections around the world [2]. Salmonella species are important food-borne pathogens responsible for disease in humans and animals [3]. They cause a wide range of human diseases such as gastroenteritis, enteric fever, and septicemia [4].

Infections caused by Salmonella spp. are increasing in Iran [5-7]. Antibiotic treatment is not required for straightforward Salmonella gastroenteritis but becomes essential to treat invasive salmonellosis $[8,9]$. Fluoroquinolones are the drugs of choice for an invasive extra intestinal infection in adults, whereas extended-spectrum cephalosporins (ESCs)

*Address correspondence to this author at the Molecular Biology Research Center, Baqiyatallah University of Medical Sciences, Tehran, Iran;

Tel: +98-21-88039883; Fax: +98-21-88039883;

E-mail: aliahmadi1345@gmail.com such as ceftazidime, cefotaxime, and ceftriaxone are drugs of choice for treatment of salmonellosis in children [10]. However, the use of these drugs is becoming compromised by the increasing development of ESCs and quinolone resistance all over the world. Salmonella strains resistant to ESC have been reported since the late 1980s, and their numbers have increased ever since.

Treatment of salmonellosis is of particular concern among children, because fluoroquinolones should not be used in this age group [11]. Treatment failures due to in vivo acquisition of an extended-spectrum $\beta$-lactamase (ESBL) gene or a reduced susceptibility to ciprofloxacin in Salmonella are now well established $[12,13]$.

Epidemiological studies are done for the prevention and treatment of infections. The ability to distinguish isolates of Salmonella may be very important to trace the source of outbreaks. The methods that have been used for deciphering the relatedness amongst the isolates are biotyping, antibiogram, plasmid typing, phage typing, serotyping, ribotyping and 
PCR fingerprinting. Some of the above techniques have low discriminatory potential, whereas others demand considerable amount of expertise, time and equipment.

In recent years, many DNA-based genotyping techniques have been utilized to delineate epidemiological relationships between various isolates [14-19]. Many reports are now available demonstrating the utility of PCR for typing of many organisms, including Salmonella [20-22]. The application of PCR based techniques has had a revolutionary impact in the diagnosis and epidemiology of infectious diseases. Utility of commonly used primers, and ERIC sequence elements in typing and differentiation of Salmonella isolates has been reported earlier [23, 24].

The ERIC sequences, also known as intergenic repeat units (IRUs), are present in many copies in genomes of members of the family [23]. The length of these elements is $126 \mathrm{bp}$ and they are highly conserved at nucleotide level and also include a central core inverted repeat. The position of ERIC elements in enterobacterial genomes varies between different species and has been used as a genetic marker to characterize isolates within a bacterial species [25, 26].

ERIC sequence was first described in Escherichia coli, Salmonella Typhimurium and other enterobacteria [23].This method uses consensus primers to amplify DNA sequences located between successive repetitive elements for subtyping Gram-negative enteric bacteria such as Salmonella spp. [23].

The objective of this study was to determine genetic relationship between extended-spectrum cephalosporins resistant S. enterica strains using ERIC-PCR.

\section{MATERIALS AND METHODS}

The study included all Salmonella isolates recovered from clinical cases clinical cases in a pediatric hospital in Tehran, during 2006-2009. The isolates had been identified by the conventional biochemical methods and serotyped by slide agglutination with commercial antisera (Staten Serum Institut, Copenhagen, Denmark).

Antimicrobial drug resistance was determined by using the disc diffusion method on Muller-Hinton agar (Merck Ltd, Darmstadt, Germany), according to the Clinical and Laboratory Standards Institute's recommendations [27] using disks (Oxoid, Basingstoke, Hampshire, United Kingdom) includ-

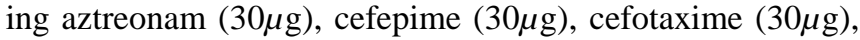
ceftazidime $(30 \mu \mathrm{g})$, ceftizoxime $(30 \mu \mathrm{g})$, ceftriaxone $(30 \mu \mathrm{g})$, and cephalothin $(30 \mu \mathrm{g})$.

Phenotypic confirmation of extended-spectrum cephalosporins resistant Salmonella was determined by the double disk synergy test using both cefotaxime and ceftazidime alone and in combination with clavulanic acid (Mast Group Ltd., Merseyside, UK). The 0.5 McFarland turbidity of bacteria were spread on the surface of MullerHinton agar. Double disk synergy test was performed by comparing the inhibition zone of disks containing cefotaxime or ceftazidime with and without clavulanic acid. The organisms used for quality control were Escherichia coli (ATCC 25922; American Type Culture Collection).

The relationship between strains was determined using ERIC-PCR. Genomic DNA was extracted as described previously [28]. The PCR amplifications were conducted by adding a mixture $(20 \mu \mathrm{l}$ per reaction) of $12 \mu \mathrm{l}$ of sterile distilled water, $2 \mu \mathrm{l}$ of $10 x$ PCR buffer, $1 \mu \mathrm{l}$ of $50 \mathrm{mM} \mathrm{MgCl}_{2}, 1$ $\mu \mathrm{l}$ of $10 \mathrm{mM}$ dNTPs, $1 \mu \mathrm{l}$ of each of primers, ERIC 1R: 5 ATG TAA GCT CCT GGG GAT TCA-3' and ERIC 2: 5'AAG TAA GTG ACT GGG GTG AGC G-3' (25), $1 \mu \mathrm{l}$ of Taq polymerase and $1 \mu \mathrm{l}$ of the template DNA to the reaction. ERIC-PCR was done as described previously $[28,29]$ with some modifications in temperature profile: An initial denaturation at $94^{\circ} \mathrm{C}$ for 4 minutes was followed by $35 \mathrm{cy}-$ cles of $94^{\circ} \mathrm{C}$ for 1 minute, annealing at $52^{\circ} \mathrm{C}$ for 1 minute, extension period at $65^{\circ} \mathrm{C}$ for 8 minutes, and a final extension at $65^{\circ} \mathrm{C}$ for 15 minutes.

Amplified products were separated by electrophoresis on $1 \%$ agarose gel and photographed under uv illumination. A $100 \mathrm{kbp}$ DNA ladder was used as the molecular size marker.

\section{RESULTS}

One hundred-thirty-six S. enterica isolates had been recovered from children in a major pediatric hospital in Tehran, during 2006-2009. All extended-spectrum cephalosporins resistant strains were selected for further analysis by ERIC-PCR in order to assess an epidemiological link among the isolates.

As shown in Table 1, six isolates including four $S$. enterica serotype Infantis and two $S$. enterica serotype

Table 1. Characteristics of the Extended-spectrum Cephalosporins Resistant Salmonella enterica Serotypes Enteritidis and Infantis

\begin{tabular}{|c|c|c|c|l|}
\hline Strains & Salmonella Serotype & Sex of Patient & ERIC Pattern & \multicolumn{1}{|c|}{ Resistance Pattern ${ }^{1)}$} \\
\hline \hline 1 & Enteritidis & Male & E1 & AM, PIP, TIC, CF, CR, CX, CZ, FE, CT \\
\hline 2 & Enteritidis & Female & E1 & AM, PIP, TIC, CF, CR, CX, CZ, CT, SXT, S, TE, NA \\
\hline 3 & Infantis & Male & E2 & AM, PIP, TIC, CF, AT, CR, CX, CZ, CT, SXT, S, TE, NA \\
\hline 4 & Infantis & Female & E2 & AM, PIP, TIC, CF, AT, CR, CX, CZ, CT, SXT, S, K, TE, NA \\
\hline 5 & Infantis & ND & E2 & AM, PIP, TIC, CF, AT, CR, CX, CZ, CT, SXT, S, TE, NA \\
\hline 6 & Infantis & Female & E2 & AM, PIP, TIC, CF, AT, CR, CX, CZ, CT, SXT, S, TE, NA \\
\hline
\end{tabular}

${ }^{11}$ AM: Ampicillin, CF: Cephalothin, CR: Ceftriaxone, CX: Cefotaxime, CZ: Ceftazidime, CT: Ceftizoxime, K: Kanamycin, NA: Nalidixic Acid, PIP: Piperacillin, SXT: Sulfamethoxazole-trimethoprim, TIC: Ticarcillin, S: Streptomycin, TET: Tetracycline.

ND, not determined 
Enteritidis showed an extended-spectrum cephalosporins resistant phenotype (i.e. simultaneous resistant to ceftriaxone, cefotaxime, and ceftazidime). Each $S$. enterica serotype Enteritidis isolates were recovered from one female and one male pediatric patient and three strains of $S$. enterica serotype Infantis were belonged to 2 female and one male patient. The sex of the patient from which one of the isolates came from was not determined.

ERIC-PCR differentiated S. enterica serotypes Infantis and Enteritidis into 2 distinct clusters. The number of ERICPCR bands produced by PCR ranged from 6 to 17, with sizes ranging from 200 to 3200 bp. ERIC-PCR patterns were arbitrarily named as two different profiles (E1 and E2). Profile E1 was found in two $S$. enterica serotype Enteritidis isolates, and profile E2 was found in four S. enterica serotype Infantis isolates (Fig. 1).

\section{DISCUSSION}

Infections caused by Salmonella spp. are endemic in Iran [30, 31]. Salmonella enterica serotypes Enteritidis and Infantis are among the most prevalent serotypes of salmonellae in humans in Iran [32-34]. Salmonella strains resistant to extended-spectrum cephalosporins are noteworthy because extended-spectrum cephalosporins are the most commonly used antimicrobial agent for the treatment of Salmonella infections in children in our country.

The strains of Salmonella spp. are usually defined phenotypically by serotyping and antimicrobial resistance tests in microbiology laboratories of Iran. Since phenotyping data suggest the clonal diffusion of a limited number of local strains, there is a need for practical techniques to discriminate Salmonella isolates, and to evaluate the association of

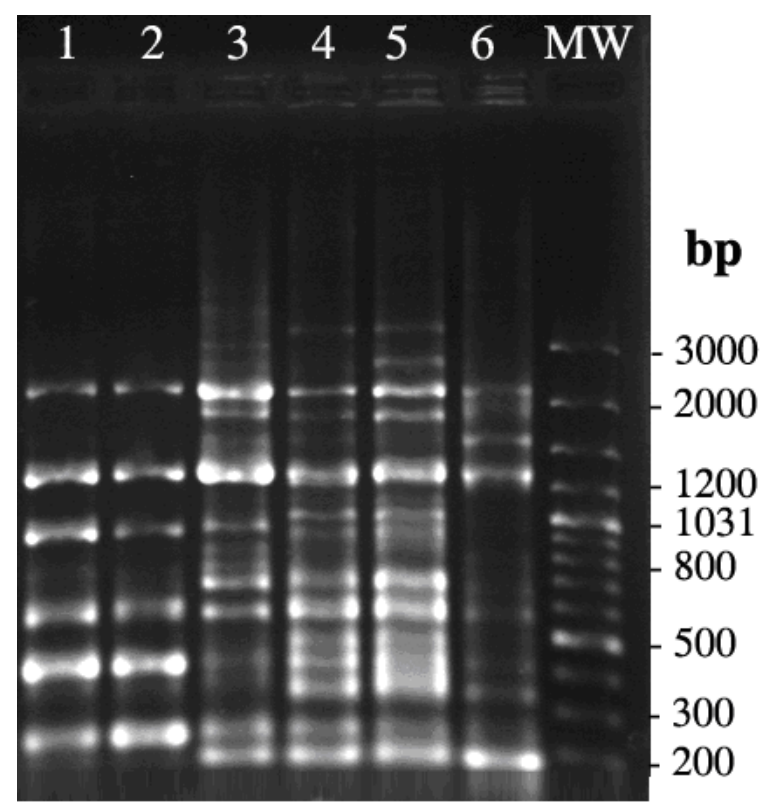

Fig. (1). Electrophoresis of ERIC-PCR products on 1\% agarose gel stained with ethidium bromide. Lanes 1 and 2 are amplification products from DNA of Salmonella enterica serotype Enteritidis, lane 3, 4, 5, and 6 are amplification products from DNA of Salmonella enterica serotype Infantis. Lane MW is 100 bp molecular size marker.
Salmonella strains in epidemiological studies [35]. Here, we presented the results of a molecular study of extendedspectrum cephalosporins resistant Salmonella strains isolated from pediatric patients in Iran. We performed ERIC-PCR using ERIC primers and obtained distinguishable DNA band patterns for each $S$. enterica serotypes including Enteritidis and Infantis.

The results indicated that ERIC-PCR could differentiate the $S$. enterica isolates at serotype level. However all strains within each serotype of Enteritidis and Infantis have shown similar ERIC-PCR pattern.

Application of ERIC-PCR fingerprinting for genotyping $S$. enterica strains has been reported in South Korea by Lim et al. [36]. They identified 50 genotypes among 57 Salmonella strains. Oliveira et al. have also reported some similar observations [24].

Our result is consistent with findings of Van Lith [37], who found each serotype was characterized by a unique DNA profile however Burr et al. characterized Salmonella serotypes and found that every isolate had a unique fingerprinting but the serotypes were not grouped together in major branches, thus, serotypes were not identified by ERIC-PCR [38].

The greatest advantages of genotyping by ERIC-PCR lie in its accessibility, speed, relative ease of use, and general stability. Unfortunately, ERIC-PCR does not always offer a complete picture of genetic relatedness. Identical bands are based on their size, and not necessarily their genetic makeup. It is possible that two genetically diverse segments between two ERIC segments can be counted as equal, provided that the ERIC targets are the same distance apart on DNA.

In conclusion, our results suggest that extended-spectrum cephalosporin resistant Salmonella could be attributed to a few predominant serotypes including Enteritidis and Infantis in this study. Genetic analysis using ERIC-PCR showed that closely related clones are responsible for the occurrence of extended spectrum cephalosporin-resistant Salmonella infection in Tehran.

\section{REFERENCES}

[1] Bennasar A, de Luna G, Cabrer B, et al. Rapid identification of Salmonella typhimurium, S. enteritidis and S. virchow isolates by polymerase chain reaction based fingerprinting methods. Int Microbiol 2000; 3: 31-8.

[2] Rasschaert G, Houf K, Imberechts H, et al. Comparison of five repetitive-sequence-based PCR typing methods for molecular discrimination of Salmonella enterica isolates. J Clin Microbiol 2005; 43: 3615-23.

[3] Albufera U, Bhugaloo-Vial P, Issack MI, et al. Molecular characterization of Salmonella isolates by REP-PCR and RAPD analysis. Infect Genet Evol 2009; 9: 322-7.

[4] Bertrand S, Weill FX, Cloeckaert A, et al. Clonal emergence of extended-spectrum $\beta$-lactamase (CTX-M-2)-producing Salmonella enterica serovar Virchow isolates with reduced susceptibilities to ciprofloxacin among poultry and humans in Belgium and France (2000 to 2003). J Clin Microbiol 2006; 44: 2897-903.

[5] Ranjbar R, Salimkhani E, Sadeghifard N, et al. An outbreak of gastroenteritis of unknown origin in Tehran, July 2003. Pak J Biol Sci 2007; 10: 1138-40.

[6] Ranjbar R, Giammanco GM, Aleo A, et al. Characterization of the first extended-spectrum beta-lactamase-producing nontyphoidal Salmonella strains isolated in Tehran, Iran. Foodborne Pathog Dis 2010; 7: 91-5. 
[7] Ranjbar R, Giammanco GM, Farshad S, et al. Serotypes, antibiotic resistance, and class 1 integrons in Salmonella isolates from pediatric cases of enteritis in Tehran, Iran. Foodborne Pathog Dis 2011; 8: 547-53.

[8] Moss P. Infections of the gastrointestinal tract. In: Finch RG, Greenwood D, Norrby RS, Whitley RJ, Eds. Antibiotics and chemotherapy: anti-infective agents and their use in therapy. Kent, Livingstone. 2003; pp. 667-81.

[9] Gilbert DN, Moellering RC, Sande MA, Eliopoulos GM. The Sanford Guide to Antimicrobial Therapy. 36th ed. Sperryville Va, 2006.

[10] Fey PD, Safranek TJ, Rupp ME, et al. Ceftriaxone-resistant Salmonella infection acquired by a child from cattle. N Engl J Med 2000; 342: 1242-9.

[11] Yates C, Amyes S. Extended-spectrum beta-lactamases in nontyphoidal Salmonella spp. isolated in the UK are now a reality: why the late arrival? J Antimicrob Chemother 2005; 56: 262-4.

[12] Su LH, Chiu CH, Chu C, et al. In vivo acquisition of ceftriaxone resistance in Salmonella enterica serotype Anatum. Antimicrob Agents Chemother 2003; 47: 563-7.

[13] Aarestrup FM, Wiuff C, Molbak K, et al. Is it time to change fluoroquinolone breakpoints for Salmonella spp.? Antimicrob Agents Chemother 2003; 47: 827-9.

[14] Kostman JR, Edlind TD, LiPuma JJ, et al. Molecular epidemiology of Pseudomonas cepacia determined by polymerase chain reaction ribotyping. J Clin Microbiol 1992; 30: 2084-7.

[15] Kerouanton A, Brisabois A, Grout J, et al. Molecular epidemiological tools for Salmonella Dublin typing. FEMS Immunol Med Microbiol 1996; 14: 25-9.

[16] Millemann Y, Lesage-Descauses MC, Lafont JP, et al. Comparison of random amplified polymorphic DNA analysis and Enterobacterial Repetitive Intergenic Consensus- PCR for epidemiological studies of Salmonella. FEMS Immunol Med Microbiol 1996; 14: 129-34.

[17] Martin MC, Gonzalez-Hevia MA, Moro I, et al. Genetic typing methods applied to the differentiation of clonal lines among Salmonella enterica serogroup $\mathrm{G}$ strains causing human salmonellosis. FEMS Immunol Med Microbiol 1997; 19: 215-21.

[18] Giovannacci I, Ragimbeau C, Queguiner S, et al. Listeria monocytogenes in pork slaughtering and cutting plants; use of RAPD, PFGE and PCR-REA for tracing and molecular epidemiology. Int J Food Microbiol 1999; 53: 127-40.

[19] Tsen H, Hu HH, Lin JS, et al. Analysis of the Salmonella typhimurium isolates from food poisoning cases by molecular subtyping methods. Food Microbiol 2000; 17: 143-52.

[20] Ménard C, Brousseau R, Mouton C. Application of polymerase chain reaction with arbitrary primer (AP-PCR) to strain identification of Porphyromonas (Bacteroides) gingivalis. FEMS Microbiol Lett 1992; 95: 163-8.

[21] Niesters HG, Goesseus WH, Meis JF et al. Rapid, polymerase chain reaction-based identification assays for Candida species. J Clin Microbiol 1993; 31: 904-10.

[22] Struelens HJ, Bax R, Deplano A, et al. Concordant clonal delineation of methicillin-resistant Staphylococcus aureus by macrorestriction analysis and polymerase chain reaction genome fingerprinting. J Clin Microbiol 1993; 31: 1964-70.

[23] Hulton CS, Higgins CF, Sharp PM. ERIC sequences: a novel family of repetitive elements in the genomes of Escherichia coli, Salmonella Typhimurium and other enterobacteria. Mol Microbiol 1991; 5: 825-34.

[24] Oliveira SD, Bessa MC, Santos LR, et al. Phenotypic and genotypic characterization of salmonella enteritidis isolates. Brazilian J Microbiol 2007; 38: 720-8.

[25] Versalovic J, Koueth T, Lupski JR. Distribution of repetitive DNA sequences in eubacteria and application to fingerprinting of bacterial genomes. Nucleic Acids Res 1991; 19: 6823-31.

[26] Radu S, Vincent M, Apun K, et al. Molecular characterization of Vibrio cholerae O1 outbreak strains in Miri, Sarawak (Malaysia). Acta Tropica 2002; 83: 169-76.

[27] Clinical and Laboratory Standards Institute: Performance Standards for Antimicrobial Susceptibility Testing. 15th Informational Supplement. Approved Standard M100-S15. Clinical and Laboratory Standards Institute, Wayne, Pa 2005.

[28] Ranjbar R, Hosseini MJ, Kaffashian AR, et al. An outbreak of shigellosis due to Shigella flexneri serotype $3 a$ in a prison in Iran. Arch Iran Med 2010; 13: 413-6.

[29] Ranjbar R, Rahbar M, Naghoni A, et al. A cholera outbreak associated with drinking contaminated well water. Arch Iran Med 2011; 14: 339-40.

[30] Ranjbar R, Mirzaee A. Determining of the variety of genotypes in Salmonella typhimurium by ERIC-PCR. J Babol Univ Med Sci 2012; 15: 51-7.

[31] Ranjbar R, Sarshar M. Genetic diversity of clinical strains of Salmonella enterica serovar Typhimurium. J Mil Med 2012; 14: 1437.

[32] Naghoni A, Ranjbar R, Tabaraie B, et al. High prevalence of integron mediated resistances among clinical isolates of Salmonella enterica strains. Jpn J Infect Dis 2010; 63: 417-21.

[33] Ranjbar R, Reza T, Mirzaee A. Molecular typing of Salmonella enteritidis strains isolated from several laboratory centers in Tehran by ERIC-PCR. SJKU 2013; 18: 77-85.

[34] Ranjbar R, Sarshar M, Morovvati S. A study of ribotype patterns of Salmonella enterica serovar Enteritidis strains isolated in Tehran, Iran. J Isfahan Med Sch 2012; 30: 238-47.

[35] Erdem B, Threlfall EJ, Schofield SL, et al. Plasmid profile typing provides a method for the differentiation of strains of Salmonella enteritidis phage type 4 isolated in Turkey. Lett Appl Microbiol 1994; 19: 265-7.

[36] Lim H, Lee KH, Hong $\mathrm{CH}$, et al. Comparison of four molecular typing methods for the differentiation of Salmonella spp. Int J Food Microbiol 2005; 105: 411-8.

[37] Van Lith LA, Aarts HJ. Polymerase chain reaction identification of Salmonella serotypes. Lett Appl Microbiol 1994; 19: 273-6.

[38] Burr MD, Josephson KL, Pepper IL. An evaluation of ERIC PCR and AP PCR fingerprinting for discriminating Salmonella serotypes. Lett Appl Microbiol 1998; 27: 24-30.

(C) Ranjbar et al.; Licensee Bentham Open.

This is an open access article licensed under the terms of the Creative Commons Attribution Non-Commercial License (http://creativecommons.org/licenses/by-nc/3.0/) which permits unrestricted, non-commercial use, distribution and reproduction in any medium, provided the work is properly cited. 